\title{
PARTIALLY BOUNDED $J$-FRACTIONS $\left({ }^{1}\right)$
}

BY

\author{
LESTER G. RIGGS AND W. T. SCOTT
}

1. Introduction. The theory of positive-definite $J$-fractions was used by Wall [3] to show that there is associated with each bounded $J$-fraction a convex region $R$, and that the $J$-fraction converges in the exterior of $R$ to an analytic function. The principal purpose of the present paper is to obtain an analog of Wall's results for a wider class of $J$-fractions whose coefficients are not necessarily bounded.

The lower bound of a $J$-form is defined in $\S 2$ and some elementary properties are noted for reference. In $\S 3$ the partial bound, $Y(\theta)$, of a $J$-fraction is defined, and necessary conditions and sufficient conditions for partial boundness are given. Also, the structure of $\theta$-sets of partial boundeness is determined, and it is shown that $Y(\theta)$ is continuous and has left and right hand derivatives.

In $\S 4$ the convex set $R$ associated with a partially bounded $J$-fraction is defined and some convergence theorems are given. Partially bounded $J$-fractions having a linear convex set $R$ are characterized, and the notion of the convex hull of a partially bounded $J$-fraction is used to extend the previously stated convergence results.

2. The lower bound and lower limit of a J-form. For two given infinite sequences of real numbers $\alpha_{1}, \alpha_{2}, \cdots$, and $\beta_{1} \beta_{2}, \cdots$, consider the quadratic forms (Jacobi forms, or $J$-forms)

$$
J_{m}^{(n)}(x, y)=\sum_{p=0}^{m-1}\left(\beta_{p+n}-y\right) x_{p+n}^{2}-2 \sum_{p=0}^{m-2} \alpha_{p+n} x_{p+n} x_{p+n+1}, \quad(m, n=1,2, \cdots),
$$

in the real variables $x_{1}, x_{2}, \cdots$, where $y$ is a real parameter. The largest value $Y_{m}^{(n)}$ of $y$ for which $J_{m}^{(n)}(x, y)$ is non-negative-definite is called the lower bound of $J^{(n)}(x, 0)$. If $M_{m}^{(n)}$ denotes the maximum of $\left|\alpha_{p+n}\right|$ and $\left|\beta_{p+n}\right|$ for $0 \leqq p \leqq m-1$ it is easily seen that $Y_{m}^{(n)} \geqq-3 M_{m}^{(n)}$ since

$$
\begin{aligned}
& J_{m}^{(n)}\left(x,-3 M_{m}^{(n)}\right) \\
& \geqq M_{m}^{(n)}\left[\left|x_{n}\right|^{2}+\left(\left|x_{n}\right|-\left|x_{n+1}\right|\right)^{2}+\cdots+\left(\left|x_{n+m-2}\right|-x_{n+m-1} \mid\right)^{2}+\left|x_{n+m-1}\right|^{2}\right] .
\end{aligned}
$$

Received by the editors October 10, 1961.

(1) The research of one of the authors was supported, in part, by the United States Air Force Office of Scientific Research under Grant No. AF-AFOSR-62-310. 
If for a fixed value of $n$ there exists a largest value $Y^{(n)}$ of $y$ for which all of the forms $J_{m}^{(n)}(x, y),(m=1,2, \cdots)$, are non-negative-definite, then the forms $J_{m}^{(n)}(x, 0)$, $(m=1,2, \cdots)$, are said to be bounded below and $Y^{(n)}$ is called their lower bound. From a result of Wall and Wetzel [5] on non-negative-definite $J$-forms it follows that the forms $J_{m}^{(n)}(x, 0),(m=1,2, \cdots)$, have lower bound $Y^{(n)}$ if and only if $Y^{(n)}$ is the largest value of $y$ which satisfies both of the following conditions:

(i) $\beta_{n+p}-y \geqq 0, \quad(p=0,1,2, \cdots)$.

(ii) There exist numbers $g_{p}, 0 \leqq g_{p} \leqq 1$, such that

$$
\alpha_{n+p}^{2} \leqq\left(\beta_{n+p}-y\right)\left(\beta_{n+p+1}-y\right)\left(1-g_{p}\right) g_{p+1}, \quad(p=0,1,2, \cdots) .
$$

The observation that $J_{m+1}^{(n)}(x, y)$ is identical with $J_{m}^{(n)}(x, y)$ when $x_{n+m}=0$ yields the inequality

$$
Y_{m+1}^{(n)} \leqq Y_{m}^{(n)}
$$

from which the following theorem is obtained.

THEOREM 2.1. The forms $J_{m}^{(n)}(x, 0),(m=1,2, \cdots)$, are bounded below with lower bound $Y^{(n)}$ if and only if

$$
\lim _{m=\infty} Y_{m}^{(n)}=Y^{(n)}
$$

TheOREM 2.2. A necessary condition for the forms $J_{m}^{(n)}(x, 0),(m=1,2, \cdots)$, to have the lower bound $Y^{(n)}$ is that

$$
B^{(n)}=\underset{0 \leqq p<\infty}{\operatorname{glb}} \beta_{n+p} \geqq Y^{(n)}
$$

A sufficient condition for the forms to be bounded below is the existence of the bound $B^{(n)}$ and of

$$
A^{(n)}=\operatorname{lub}_{0 \leqq p<\infty}\left|\alpha_{n+p}\right|
$$

in which case the lower bound $Y^{(n)}$ satisfies

$$
B^{(n)}-2 A^{(n)} \leqq Y^{(n)} \leqq B^{(n)}
$$

The stated necessary condition is an immediate consequence of (i) of (2.1). The sufficiency of (2.4) and (2.5), and the validity of inequality (2.6), follow by noting that (2.1) holds for $g_{p}=1 / 2$ when $y=B^{(n)}-2 A^{(n)}$.

The observation that $J_{m+1}^{(n)}(x, y)$ is identical with $J_{m}^{(n+1)}(x, y)$ when $x_{n}=0$ yields the inequality 


$$
Y_{m+1}^{(n)} \leqq Y_{m}^{(n+1)}
$$

Also, the decomposition

$$
J_{m+1}^{(n)}(x, y)=J_{m}^{(n+1)}(x, y)+J_{2}^{(n)}(x, y)-J_{1}^{(n+1)}(x, y)
$$

and the fact that

$$
\begin{aligned}
& Y_{m}^{(n+1)}+Y_{2}^{(n)}-Y_{1}^{(n+1)}=\min \left\{Y_{m}^{(n+1)}, Y_{2}^{(n)}, Y_{m}^{(n+1)}+Y_{2}^{(n)}-Y_{1}^{(n+1)}\right\}, \\
& J_{m+1}^{(n)}(x, y) \geqq 0 \text { for } y=Y_{m}^{(n+1)}+Y_{2}^{(n)}-Y_{1}^{(n+1)}
\end{aligned}
$$

lead to the inequality

$$
Y_{m+1}^{(n)} \geqq Y_{m}^{(n+1)}+Y_{2}^{(n)}-Y_{1}^{(n+1)}
$$

The following result is a consequence of (2.7) and (2.8).

THEOREM 2.3. If $Y^{(n)}$ exists for a single index $n$, it exists for $n=1,2, \cdots$, and

$$
Y^{(n+1)}+Y_{2}^{(n)}-Y_{1}^{(n)} \leqq Y^{(n)} \leqq Y^{(n+1)}
$$

If

$$
Y^{(\infty)}=\lim _{n=\infty} Y^{(n)}
$$

is finite, then the forms $J_{m}^{(n)}(x, y)$ are said to have the lower limit $Y^{(\infty)}$.

3. The partial bound of a $J$-fraction. A $J$-fraction is a continued fraction of the form

$$
K(z)=K_{1}(z)=\frac{1}{z+b_{1}}-\frac{a_{1}^{2}}{z+b_{2}}-\frac{a_{2}^{2}}{z+b_{3}}-\cdots
$$

in which $a_{p}, b_{p}$ are complex numbers and $a_{p} \neq 0, p=1,2, \cdots$, (nonterminating), or else $a_{p} \neq 0, p \leqq k, a_{p}=0, p>k$, (terminating). $K(z)$ is said to be positive definite for $\operatorname{Im} z>0$ provided the forms $J_{m}(x, 0)=J_{m}^{(1)}(x, 0)$ of $\S 2$ are nonnegative-definite, where

$$
a_{p}=s_{p}+i \alpha_{p}, \quad b_{p}=t_{p}+i \beta_{p},
$$

(cf. Wall and Wetzel [4, p.375]). If the forms $J_{m}(x, 0)$ have lower bound $Y=Y^{(1)}$, $K(z)$ is said to be bounded below with lower bound $Y$. An equivalent statement is that $K(z-i \mathrm{Y})$ is positive-definite for $\operatorname{Im} z>0$, or that $K(z)$ is positive-definite for $\operatorname{Im} z>-Y$.

In his study of bounded $J$-fractions Wall [3] introduced an arbitrary real parameter into the iproblem by noting that a simple equivalence transformation of $K(z)$ yields the new $J$-fraction in $\zeta=e^{i \theta} z$, 


$$
e^{-i \theta} K(z)=\frac{1}{\zeta+e^{i \theta} b_{1}}-\frac{\left(e^{i \theta} a_{1}\right)^{2}}{\zeta+e^{i \theta} b_{2}}-\frac{\left(e^{i \theta} a_{2}\right)^{2}}{\zeta+e^{i \theta} b_{3}}-\cdots .
$$

For this $J$-fraction the roles of $\alpha_{p}$ and $\beta_{p}$ in the related $J$-forms are assumed by

$$
\alpha_{p}(\theta)=\operatorname{Im} e^{i \theta} a_{p}, \quad \beta_{p}(\theta)=\operatorname{Im} e^{i \theta} b_{p} .
$$

If for each $\theta$ in a set $T, e^{-i \theta} K(z)$ is bounded below with lower bound $Y(\theta)$, then $K(z)$ is said to be partially bounded, with partial bound $Y(\theta)$, for $\theta$ in $T$. In view of the periodicity of $e^{i \theta}$, it is clear that $T$ will be completely determined by a knowledge of the part of $T$ in any half-open $\theta$ interval of length $2 \pi$.

The remainder of this section is devoted to a determination of the structure of a set $T$ of partial boundeness, and to a study of some properties of the partial bound, $Y(\theta)$, on $T$.

THEOREM 3.1. If $K(z)$ has partial bound $Y(\theta)$ for $\theta_{1}<\theta<\theta_{2}$ then

$$
B(\theta)=\underset{1 \leqq p<\infty}{\operatorname{glb}} \beta_{p}(\theta) \geqq Y(\theta), \quad \theta_{1}<\theta<\theta_{2},
$$

and the elements $b_{p},(p=1,2, \ldots)$, lie in the convex region $\mathscr{B}$ of the $b$-plane $(b=t+i \beta)$ defined $b y$

$$
\mathscr{B}: \beta(\theta) \geqq B(\theta), \quad \theta_{1}<\theta<\theta_{2},
$$

where $\beta(\theta)=\operatorname{Im} e^{i \theta} b$. A sufficient condition for the partial boundedness of $K(z)$ for $\theta_{1}<\theta<\theta_{2}$ is the existence of $B(\theta)$ and of

$$
A(\theta)=\operatorname{lub}_{1 \leqq p<\infty}\left|\alpha_{p}(\theta)\right|, \quad \theta_{1}<\theta<\theta_{2},
$$

in which case the elements $a_{p},(p=1,2, \cdots)$, are bounded and the partial bound, $Y(\theta)$, of $K(z)$ satisfies

$$
B(\theta)-2 A(\theta) \leqq Y(\theta) \leqq B(\theta), \quad \theta_{1}<\theta<\theta_{2} .
$$

Inequality (3.6) and the statements of both necessary and sufficient conditions follow at once from Theorem 2.2 and the definition of partial boundedness. The remark on the boundedness of the elements $a_{p}$ is easily verified by noting that the existence of $A(\theta)$ for any two distinct values of $\theta$ differing by less than $\pi$ implies boundedness for the real and imaginary parts of $a_{p}$.

In order to show that the region $\mathscr{B}$ of (3.4) is convex it is sufficient to observe that any point $b$ on the line joining points $b^{\prime}$ and $b^{\prime \prime}$ of $\mathscr{B}$ has the representation

$$
b=\sigma b^{\prime}+(1-\sigma) b^{\prime \prime}
$$

where $0 \leqq \sigma \leqq 1$. Then for all $\theta, \theta_{1}<\theta<\theta_{2}$,

$$
\beta(\theta)=\sigma \beta^{\prime}(\theta)+(1-\sigma) \beta^{\prime \prime}(\theta) \geqq B(\theta),
$$

and $b$ is in the region $\mathscr{B}$. 
This completes the proof of Theorem 3.1.

For the bounded $J$-fractions of Wall [2] the elements $a_{p}$ and $b_{p}$ are bounded. Theorem 3.1 shows that a bounded $J$-fraction is partially bounded for $0 \leqq \theta \leqq 2 \pi$ and the partial bound in this case is identical with the bound defined by Wall. The following theorem gives a condition under which a partially bounded $J$-fraction will be a bounded $J$-fraction.

THEOREM 3.2. If $K(z)$ is partially bounded for $\theta_{1}<\theta<\theta_{2}$, where $\theta_{2}-\theta_{1}>\pi$, then $K(z)$ is bounded.

The boundedness of the elements $a_{p}$ is a consequence of Theorem 3.1. It is easily seen that the elements $b_{p}$ are bounded since the hypothesis insures the existence if $Y(\theta)$ for the four distinct $\theta$-values, $\theta^{\prime}, \theta^{\prime}+\pi, \theta^{\prime \prime}, \theta^{\prime \prime}+\pi$, where $0<\theta^{\prime \prime}-\theta^{\prime}<\pi$ (i.e., the convex region $\mathscr{B}$ is bounded since its lines of support turn through an angle greater than $\pi$ ).

THEOREM 3.3. If $K(z)$ is partially bounded for $\theta=\theta_{0}, \theta_{0}+\pi$, then

$$
Y\left(\theta_{0}\right)+Y\left(\theta_{0}+\pi\right) \leqq 0 .
$$

The proof is a consequence of Theorem 3.1 since $\beta_{p}\left(\theta_{0}+\pi\right)=-\beta_{p}\left(\theta_{0}\right)$.

THEOREM 3.4. If $K(z)$ is partially bounded in $T$ and $\theta_{1} \in T, \theta_{2} \in T$, where $0<\theta_{2}-\theta_{1}<\pi$, then $T$ contains the interval $\theta_{1} \leqq \theta \leqq \theta_{2}$, and the partial bound of $K(z)$ satisfies

$$
Y(\theta) \geqq \frac{\sin \left(\theta_{2}-\theta\right)}{\sin \left(\theta_{2}-\theta_{1}\right)} Y\left(\theta_{1}\right)+\frac{\sin \left(\theta-\theta_{1}\right)}{\sin \left(\theta_{2}-\theta_{1}\right)} Y\left(\theta_{2}\right), \quad \theta_{1} \leqq \theta \leqq \theta_{2} .
$$

For any $\theta$,

$$
\begin{aligned}
& \alpha_{p}(\theta)=\frac{\sin \left(\theta_{2}-\theta\right)}{\sin \left(\theta_{2}-\theta_{1}\right)} \alpha_{p}\left(\theta_{1}\right)+\frac{\sin \left(\theta-\theta_{1}\right)}{\sin \left(\theta_{2}-\theta_{1}\right)} \alpha_{p}\left(\theta_{2}\right), \\
& \beta_{p}(\theta)=\frac{\sin \left(\theta_{2}-\theta\right)}{\sin \left(\theta_{2}-\theta_{1}\right)} \beta_{p}\left(\theta_{1}\right)+\frac{\sin \left(\theta-\theta_{1}\right)}{\sin \left(\theta_{2}-\theta_{1}\right)} \beta_{p}\left(\theta_{2}\right),
\end{aligned}
$$

and the $J$-forms associated with $e^{-i \theta} K(z)$ are

$$
\begin{aligned}
J_{m}(x, 0)= & \frac{\sin \left(\theta_{2}-\theta\right)}{\sin \left(\theta_{2}-\theta_{1}\right)}\left[\sum_{p=0}^{m-1} \beta_{p+1}\left(\theta_{1}\right) x_{p+1}^{2}-2 \sum_{p=0}^{m-2} \alpha_{p+1}\left(\theta_{1}\right) x_{p+1} x_{p+2}\right] \\
& \quad+\frac{\sin \left(\theta-\theta_{1}\right)}{\sin \left(\theta_{2}-\theta_{1}\right)}\left[\sum_{p=0}^{m-1} \beta_{p+1}\left(\theta_{2}\right) x_{p+1}^{2}-2 \sum_{p=0}^{m-2} \alpha_{p+1}\left(\theta_{2}\right) x_{p+1} x_{p+2}\right] .
\end{aligned}
$$

Since the terms in square brackets are the $J$-forms $J_{m}(x, 0)$ associated with $e^{-i \theta_{1}} K(z)$ and $e^{-i \theta_{2}} K(z)$, respectively, it follows that for $\theta_{1} \leqq \theta \leqq \theta_{2}$, 


$$
J_{m}(x, 0) \geqq\left[\frac{\sin \left(\theta_{2}-\theta\right)}{\sin \left(\theta_{2}-\theta_{1}\right)} Y_{m}\left(\theta_{1}\right)+\frac{\sin \left(\theta-\theta_{1}\right)}{\sin \left(\theta_{2}-\theta_{1}\right)} Y_{m}\left(\theta_{2}\right)\right]_{p=0}^{m-1} x_{p+n}^{2} .
$$

Thus $J_{m}(x, 0)$ has a lower bound $Y_{m}(\theta)$ which satisfies

$$
Y_{m}(\theta) \geqq \frac{\sin \left(\theta_{2}-\theta\right)}{\sin \left(\theta_{2}-\theta_{1}\right)} Y_{m}\left(\theta_{1}\right)+\frac{\sin \left(\theta-\theta_{1}\right)}{\sin \left(\theta_{2}-\theta_{1}\right)} Y_{m}\left(\theta_{2}\right) .
$$

The proof of Theorem 3.4 may now be completed by noting that this last inequality, together with (2.2) and Theorem 2.1, implies the existence of $Y(\theta)$ and the validity of (3.7).

It is easy to see that, modulo $2 \pi$, a set $T$ of partial boundedness must have one of the following forms:

(i) $T$ is vacuous;

(ii) $T$ contains a single point, $\theta_{0}$;

(iii) $T$ contains two points, $\theta_{0}$ and $\theta_{0}+\pi$;

(iv) $T$ contains a $\theta$-interval of length not exceeding $\pi$.

(v) $T$ contains a closed $\theta$-interval of length $2 \pi$.

The fact that each of these possibilities may occur is illustrated by the $J$-fractions, $K(z)$, given below.

(i) $\left|b_{p}\right| \rightarrow \infty$ as $p \rightarrow \infty$ and the numbers, $\arg b_{p}$, are everywhere dense in $[0,2 \pi]$. Then $\operatorname{glb} \beta_{p}(\theta)=-\infty$ for each $\theta$ and $K(z)$ is not partially bounded for any $\theta$.

(ii) $a_{p}$ real, $\left|b_{p}\right| \rightarrow \infty$ as $p \rightarrow \infty$ and the numbers, arg $b_{p}$, are everywhere dense in $[0, \pi]$. Reference to (2.1) shows that $Y(0)=\operatorname{glb} \beta_{n}(0) \geqq 0$, but $K(z)$ is not partially bounded for any $\theta, 0<\theta<2 \pi$, since glb $\beta_{n}(\theta)=-\infty$.

(iii) $a_{p}, b_{p}$ real, lim inf $b_{p}=-\infty, \lim \sup b_{p}=\infty$. Reference to (2.1) shows that $Y(0)=Y(\pi)=0$, but glb $\beta_{n}(\theta)=-\infty$ for $0<\theta<\pi$ and $\pi<\theta<2 \pi$.

(iv) $b_{p}>0$, lim inf $b_{p}=0$, lim sup $b_{p}=\infty$, and $a_{p}^{2}=b_{p} b_{p+1}\left(1-g_{p-1}\right) g_{p}$ where $0<g_{p-1}<1$. It can be verified from (2.1) that $Y(\theta)=0$ for $0 \leqq \theta \leqq \pi$, and $K(z)$ is not partially bounded for any $\theta, \pi<\theta<2 \pi$, since glb $\beta_{p}(\theta)=-\infty$.

(v) $K(z)$ is any bounded $J$-fraction.

Implications of the inequality (3.7), which is similar to a convexity condition on $Y(\theta)$, are given in the two following theorems. These results are stated in terms of partial bounds, but apply also to any function satisfying (3.7).

THEOREM 3.5. If $K(z)$ has partial bound $Y(\theta)$ for $\theta_{1}<\theta<\theta_{2}$ then $Y(\theta)$ is continuous for $\theta_{1}<\theta<\theta_{2}$. Moreover, the existence of either one of the limits

$$
\begin{gathered}
Y_{*}\left(\theta_{1}^{+}\right)=\underset{\theta=\theta_{1}^{+}}{\lim \inf Y(\theta),} \\
Y^{*}\left(\theta_{1}^{+}\right)=\quad \lim \sup _{\theta=\theta_{1}^{+}} Y(\theta)
\end{gathered}
$$

implies the existence of

$$
Y\left(\theta_{1}^{+}\right)=\lim _{\theta=\theta^{+}} Y(\theta)
$$


in which case $K(z)$ is partially bounded for $\theta_{1} \leqq \theta<\theta_{2}$, and $Y\left(\theta_{1}\right)=Y\left(\theta_{1}^{+}\right)$. $A$ corresponding remark applies for $\theta=\theta_{2}$.

For arbitrary $\theta, \theta_{1}<\theta<\theta_{2}$, and for $\phi>0$, sufficiently small, the relations

$$
\begin{aligned}
Y(\theta) & \geqq \frac{\sin (1-t) \phi}{\sin \phi} Y(\theta-t \phi)+\frac{\sin t \phi}{\sin \phi} Y[\theta+(1-t) \phi], \\
Y(\theta+t \phi) & \geqq \frac{\sin (1-t) \phi}{\sin \phi} Y(\theta)+\frac{\sin t \phi}{\sin \phi} Y(\theta+\phi)
\end{aligned}
$$

are obtained from (3.7) by replacing $\theta_{1}<\theta<\theta_{2}$ by $\theta-t \phi<\theta<\theta+(1-t) \phi$ and $\theta<\theta+t \phi<\theta+\phi$, respectively, where $0<t<1$. When $t \rightarrow 0^{+}$the second relation (3.8) yields $Y_{*}\left(\theta^{+}\right) \geqq Y(\theta)$ and, similarly, a statement equivalent to $Y_{*}\left(\theta^{-}\right) \geqq Y(\theta)$ is obtained when $t \rightarrow 1^{-}$. When $\phi \rightarrow 0^{+}$the first relation (3.8) yields

$$
\begin{aligned}
& Y(\theta) \geqq(1-t) Y^{*}\left(\theta^{-}\right)+t Y_{*}\left(\theta^{+}\right), \\
& Y(\theta) \geqq(1-t) Y_{*}\left(\theta^{-}\right)+t Y^{*}\left(\theta^{+}\right) .
\end{aligned}
$$

Since these relations also hold for $t=0$ and $t=1$ the continuity of $Y(\theta)$ for each $\theta, \theta_{1}<\theta<\theta_{2}$, is a consequence of the inequalities

$$
Y_{*}\left(\theta^{ \pm}\right) \geqq Y(\theta) \geqq Y^{*}\left(\theta^{ \pm}\right) .
$$

When $Y^{*}\left(\theta_{1}^{+}\right)$exists, the continuity of $Y(\theta)$ for $\theta_{1}<\theta<\theta_{2}$ insures the validity of the second relation (3.8) with $\theta=\theta_{1}$ and with $Y(\theta)$ replaced by $Y^{*}\left(\theta_{1}^{+}\right)$. The existence of $Y\left(\theta_{1}^{+}\right)$follows from the resulting inequality, $Y_{*}\left(\theta_{1}^{+}\right) \geqq Y^{*}\left(\theta_{1}^{+}\right)$, and the fact that $K(z)$ is partially bounded for $\theta_{1} \leqq \theta<\theta_{2}$ is a consequence of the continuity of $Y(\theta)$ and of the forms $J_{m}(x, 0)$ for $\theta_{1} \leqq \theta<\theta_{2}$.

The proof of Theorem 3.5 may now be completed by using a similar procedure to dispose of the cases in which one of $Y_{*}\left(\theta_{1}^{+}\right), Y^{*}\left(\theta_{2}^{-}\right), Y_{*}\left(\theta_{2}^{-}\right)$exists.

THEOREM 3.6. A partial bound, $Y(\theta)$, defined in $\theta_{1}<\theta<\theta_{2}$, has finite right and left hand derivatives for each $\theta, \theta_{1}<\theta<\theta_{2}$.

For $\phi>0$, sufficiently small, and for $0<t<1$, the second relation (3.8) gives

$$
\frac{Y(\theta+t \phi)-Y(\theta)}{t \phi} \geqq \frac{\sin t \phi Y(\theta+\phi)}{t \phi \sin \phi}+\frac{\sin (1-t) \phi-\sin \phi}{t \phi \sin \phi} Y(\theta) .
$$

As $t \rightarrow 0$ it is found that the lower right hand derivative satisfies

$$
D_{+} Y(\theta) \geqq \frac{1}{\sin \phi}[Y(\theta+\phi)-\cos \phi Y(\theta)] .
$$

As $\phi \rightarrow 0$ this yields

$$
D_{+} Y(\theta) \geqq D^{+} Y(\theta),
$$


which shows that the right hand derivative exists, finite or infinite. From the first relation (3.8),

$$
\frac{\sin \phi-\sin t \phi}{(1-t) \phi \sin t \phi} Y(\theta) \geqq \frac{\sin (1-t) \phi Y(\theta-t \phi)}{(1-t) \phi \sin t \phi}+\frac{Y[\theta+(1-t) \phi]-Y(\theta)}{(1-t) \phi} .
$$

When $t \rightarrow 1$ this shows that

$$
\frac{\cos \phi}{\sin \phi} Y(\theta) \geqq \frac{Y(\theta-\phi)}{\sin \phi}+D^{+} Y(\theta),
$$

and it follows that $D^{+} Y(\theta)=D_{+} Y(\theta)$ is finite.

A similar treatment of the upper and lower left hand derivatives will complete the proof of Theorem 3.6.

4. Convergence theorems. If a $J$-fraction $K(z)$ has partial bound $Y(\theta)$ for $\theta$ in a set $T$ of the sort determined in $\S 3$, then it is easily verified, by an argument similar to that used for the region $\mathscr{B}$ of Theorem 3.1, that the set $R$ of points in the $z$-plane defined by

$$
R: \operatorname{Im} e^{i \theta} z \leqq-Y(\theta), \quad \theta \in T,
$$

is convex. $R$ is called the convex set of $K(z)$.

The following theorem is due to Wall [3].

THEOREM 4.1. If $K(z)$ is a bounded $J$-fraction then its convex set $R$ contains the zeros of the denominators of $K(z)$, and $K(z)$ converges uniformly on each compact set in the exterior of $R$ to a function analytic in the exterior of $R$.

A theorem of Dennis and Wall [1] which will be used in the sequel is stated below in the terminology of the present paper.

THEOREM 4.2. If $K(z)$ is partially bounded for $\theta=\theta_{0}$, and if $K(z)$, or an infinite subsequence of its approximants, converges at a single point exterior to the half plane $R: \operatorname{Im} e^{i \theta_{0}} z \leqq-Y\left(\theta_{0}\right)$, then $K(z)$, or the subsequence of approximants, converges uniformly on each compact subset in the exterior of $R$ to a function analytic in the exterior of $R$.

Below is an analog of Theorem 4.1 for partially bounded $J$-fractions.

THEOREM 4.3. If $K(z)$ has partial bound $Y(\theta)$ for $\theta$ in an interval I of length not exceeding $\pi$ then the zeros of the denominators of $K(z)$ lie in the unbounded convex region $R$ defined by (4.1) for $\theta$ in I. Moreover, the approximants of $K(z)$ are uniformly bounded on each compact region at positive distance from $R$, and the convergence of $K(z)$ or a subsequence of its approximants at a single point exterior to $R$ implies uniform convergence on each such compact region to a function analytic in the exterior of $R$. 
Proof. From the definition of partial boundedness it follows that for each $\theta$ in $I, e^{-i \theta} K(z)$ has lower bound $Y(\theta)$ for $\operatorname{Im} e^{i \theta} z>0$, and hence that $e^{-i \theta} K(z)$ is positive definite for $\operatorname{Im} e^{i \theta} z>-Y(\theta)$. The $n$th approximant of $e^{-i \theta} K(z)$ is $e^{-i \theta} P_{n}(z) / Q_{n}(z)$ where $P_{n}(z) / Q_{n}(z)$ is the $n$th approximant of $K(z)$; and by a well-known property of positive definite $J$-fractions, (cf. [2, p. 113]),

$$
\left|e^{-i \theta} \frac{P_{n}(z)}{Q_{n}(z)}\right|=\left|\frac{P_{n}(z)}{Q_{n}(z)}\right| \leqq \frac{1}{\delta}
$$

holds for arbitrary $\delta>0$ provided

$$
\operatorname{Im} e^{i \theta} z \geqq-Y(\theta)+\delta, \quad \theta \text { in } I .
$$

Since the numerators, $P_{n}(z)$, and denominators, $Q_{n}(z)$, are polynomials in $z$ without common factors it is immediate that the zeros of $Q_{n}(z)$ lie in $R$.

The remark on uniform boundedness is readily verified by noting that if the minimum distance of the compact region from $T$ is denoted by $\delta$, then for each point $z$ of the region there is some $\theta$ in $I$ for which (4.3) and (4.2) hold. Thus $1 / \delta$ is a uniform bound.

If $z_{0}$ is a point exterior to $R$ at which $K(z)$ or a subsequence of its approximants converge then there is a $\theta_{0}$ in $I$ and a $\delta_{0}>0$ such that $\operatorname{Im} e^{i \theta_{0}} z_{0} \geqq-Y\left(\theta_{0}\right)+\delta_{0}$. By Theorem 4.2, after deleting a factor $e^{-i \theta_{0}}$, it is seen that $K(z)$ or its subsequence of approximants converges to a function analytic in the half plane $\operatorname{Im} e^{i \theta_{0}} z>-Y\left(\theta_{0}\right)$. The function may be continued analytically throughout the exterior of $R$ by re-applying Theorem 4.2 after noting that the half plane $\operatorname{Im} e^{i \theta} z>-Y(\theta)$ overlaps the half plane $\operatorname{Im} e^{i \theta_{0}} z>-Y\left(\theta_{0}\right)$ if $\left|\theta-\theta_{0}\right|<\pi$.

The proof of Theorem 4.3 may now be completed by using the previously established remark on uniform boundedness to verify the statement on uniform convergence.

A sufficient condition for the convergence of $K(z)$ in either Theorem 4.2 or Theorem 4.3 is the divergence of the series $\Sigma\left|a_{p}\right|^{-1}$. The divergence of the series insures the determinate case for $K(z)$, and a positive definite $J$-fraction converges when the determinate case holds $[1, \S 4 ; 2$, pp. $109 \mathrm{ff}$.$] .$

The proof of the following result is similar to that of Theorem 4.3.

THEOREM 4.4. If $K(z)$ is partially bounded for $\theta=\theta_{0}, \theta=\theta_{0}+\pi$, then the zeros of the denominators of $K(z)$ lie in the strip

$$
R: Y\left(\theta_{0}+\pi\right) \leqq \operatorname{Im} e^{i \theta_{0}} z \leqq-Y\left(\theta_{0}\right) .
$$

The approximants of $K(z)$ are uniformly bounded on each compact connected region exterior to $R$ and the convergence of $K(z)$ or a subsequence of its approximants at a single point in the half plane $\operatorname{Im} e^{i \theta_{0}} z>-Y\left(\theta_{0}\right)$ (or $\left.\operatorname{Im} e^{i \theta_{0}} z<Y\left(\theta_{0}+\pi\right)\right)$ implies uniform convergence on each compact region in that half plane to a function analytic in the half plane. 
The following theorem identifies those partially bounded $J$-fractions whose associated convex region $R$ has no interior points with real $J$-fractions, and makes available for them the convergence results for real $J$-fractions (cf. [4, pp. $114 \mathrm{ff}$.]).

THEOREM 4.5. A J-fraction $K(z)$ is partially bounded and its associated convex set $R$ is without interior points if and only if there exist real numbers $\theta_{0}$ and $y$ such that $e^{-i \theta_{0}} K(z)$ is a real $J$-fraction in $\zeta=e^{i \theta_{0}} z+i y$.

If $K(z)$ is partially bounded and $R$ has no interior points then there exists $\theta_{0}$ such that $e^{-i \theta} K(z)$ is bounded below for $\theta=\theta_{0}, \theta_{0}+\pi$, and $Y\left(\theta_{0}+\pi\right)=-Y\left(\theta_{0}\right)$. Since $e^{-i \theta_{0}} K(z)$ and $e^{-i\left(\theta_{0}+\pi\right)} K(z)$ are positive-definite for $\operatorname{Im} e^{i \theta_{0}} z>-Y\left(\theta_{0}\right)$ and for $\operatorname{Im} e^{i\left(\theta_{0}+\pi\right)} z>-Y\left(\theta_{0}+\pi\right)$, respectively, (2.1) shows that

$$
\begin{aligned}
\beta_{p}\left(\theta_{0}\right)-Y\left(\theta_{0}\right) & \geqq 0, \\
\beta_{p}\left(\theta_{0}+\pi\right)-Y\left(\theta_{0}+\pi\right) & =-\left[\beta_{p}\left(\theta_{0}\right)-Y\left(\theta_{0}\right)\right] \geqq 0,
\end{aligned}
$$

and hence that $\beta_{p}\left(\theta_{0}\right)-Y\left(\theta_{0}\right)=0, \alpha_{p}\left(\theta_{0}\right)=0$. Thus $e^{-i \theta_{0}} K(z)$ is a real $J$ fraction in $\zeta=e^{i \theta_{0}} z+i Y\left(\theta_{0}\right)$.

Conversely, if $e^{-i \theta_{0}} K(z)$ is a real $J$-fraction in $\zeta=e^{i \theta_{0}} z+i y$ then $\alpha_{p}\left(\theta_{0}\right)=0$ and $\beta_{p}(\theta)-y=0$, so that $K(z)$ is partially bounded for $\theta=\theta_{0}$ and $Y\left(\theta_{0}\right)=Y$. But also, $e^{-i\left(\theta_{0}+\pi\right)} K(z)$ is a real $J$-fraction in $\zeta=e^{i\left(\theta_{0}+\pi\right)}+i y$, and $K(z)$ is partially bounded for $\theta=\theta_{0}+\pi$ with $Y\left(\theta_{0}+\pi\right)=-y$. It follows from Theorem 4.4 that the associated convex region $R$ is contained in the line $\operatorname{Im} e^{i \theta_{0}} z=-y$.

In the terminology of $\S 2$, the preceding parts of the present section have dealt with the $J$-fraction $K(z)=K_{1}(z)$, where

$$
K_{n}(z)=\frac{1}{z+b_{n}}-\frac{a_{n}^{2}}{z+b_{n+1}}-\frac{a_{n+1}^{2}}{z+b_{n+2}}-\cdots,
$$

and with the related partial bound $Y(\theta)=Y^{(1)}(\theta)$, and convex set $R=R^{(1)}$. In view of the formal identity

$$
K(z)=\frac{P_{n}(z)-a_{n}^{2} P_{n-1}(z) K_{n+1}(z)}{Q_{n}(z)-a_{n}^{2} Q_{n-1}(z) K_{n+1}(z)},
$$

it is evident that information about the convergence of $K_{n}(z)$ yields information about the behavior of $K(z)$. To facilitate the statement of results,

$$
Y^{(\infty)}(\theta)=\lim Y^{(n)}
$$

is called the partial limit of $K(z)$ and the related convex set,

$$
R^{(\infty)}: \operatorname{Im} e^{i \theta} z \leqq-Y^{(\infty)}(\theta),
$$

is called the convex hull of $K(z)$. 
In the case of terminating $J$-fractions the machinery for the determination of $Y^{(\infty)}(\theta)$ breaks down. The difficulty can be eliminated by defining $Y_{(\theta)}^{(\infty)}$ to be $+\infty$ and $R^{(\infty)}$ to be the null set in this case. This situation will be easy to identify in terms of the convex sets $R^{(n)}$ since Theorem 4.5 and a simple computation show that $K(z)$ is terminating if and only if there is an index $n$ for which $K_{n}(z)$ has the associated set $R^{(n)}$ consisting of a single point.

Theorem 2.3 shows that $Y^{(n)}(\theta)$ exists for those and only those values of $\theta$ for which $Y(\theta)$ exists, and for such $\theta, R^{(n+1)} \subset R^{(n)}$. The identity (4.5) and the fact that a non-terminating $J$-fraction cannot converge to a rational function of $z$ (cf. [2, p. 197]) leads to the following result, which is, in a sense, an extension of Theorem 4.1.

THEOREM 4.6. If $K(z)$ is a bounded J-fraction, then $K(z)$ converges in the exterior of its convex hull, except perhaps at certain isolated points which may cluster on the boundary of $R^{(\infty)}$, to a function meromorphic in the exterior of $R^{(\infty)}$. The excepted points, if they exist, are poles of the meromorphic function, and the convergence of $K(z)$ to the function is uniform in any compact region which is exterior to $R^{(\infty)}$ and contains none of the excluded points.

For any compact region $G$ exterior to $R^{(\infty)}$, there exists an index $n$ such that $G$ is exterior to $R^{(n)}$. The proof of the theorem may be completed by applying Theorem 4.1 to the bounded $J$-fraction $K_{n}(z)$ and noting that, by (4.5), the poles of the meromorphic function in $G$ occur only at those isolated points of $G$ where $Q_{n-1}(z)-a_{n-1}^{2} Q_{n-2}(z) K_{n}(z)=0$.

The statement, in terms of the convex hull, of similar extensions of Theorems 4.2-4.4 is omitted.

It is interesting to note that the convex hull for a partially bounded $J$-fraction may be the null set. This is the case for example (ii) of $\S 3$ if $\beta_{n}(0) \rightarrow \infty$.

\section{REFERENCES}

1. J. J. Dennis and H. S. Wall, The limit-circle case for a positive definite J-fraction, Duke Math. J. 12 (1945), 255-273.

2. H. S. Wall, Analytic theory of continued fractions, Van Nostrand, New York, 1948.

3. - B Bounded J-fractions, Bull. Amer. Math. Soc. 52 (1946), 686-693.

4. H. S. Wall and Marion Wetzel, Contributions to the analytic theory of J-fractions, Trans. Amer. Math. Soc. 55 (1944), 373-397.

5. - - Quadratic forms and convergence regions for continued fractions, Duke Math. J. 11 (1944), 89-102.

San Diego State College, San Diego, California ARIzona State University, TEMPE, ARIZONA 